\title{
Challenges for Case-Control Studies with Microbiome Data
}

\author{
J. Paul Brooks ${ }^{\mathrm{a}, *}$ \\ ${ }^{a}$ Department of Statistical Sciences and Operations Research, Center for the Study of \\ Biological Complexity, Department of Supply Chain Management and Analytics, Virginia \\ Commonwealth University, Richmond, VA
}

\begin{abstract}
In case-control studies of the human microbiome, the objective is often to determine (1) if cases differ from controls in the microbiome composition of a particular body habitat, and (2) which taxa are responsible for the differences. These studies leverage sequencing technology and spectroscopy that provide new measurements of the microbiome. Labs make choices about measurement protocols based on their environment of interest and the questions they wish to answer. Understanding the effects of these choices on case-control analyses has been underappreciated, with an implicit assumption that further advances in technology will address all of the current shortcomings. I propose several recommendations, including positive and negative control experiments to provide insight into bias, contamination, and technical variation. These experiments can help investigators make quantitative and qualitative adjustments to data in order to prevent false discoveries, increase power to discover true disease determinants, and enhance interpretation across studies.
\end{abstract}

Wordcount: 3723

Keywords: microbiome, control experiments, rare taxa, bias, normalization

\footnotetext{
*Corresponding author

Email address: jpbrooks@vcu.edu (J. Paul Brooks)

Preprint submitted to Annals of Epidemiology

January 25, 2016
} 


\section{Introduction}

Advances in sequencing technology and spectroscopy allow deeper insight into the composition and function of the human microbiome. New findings may have epidemiological and clinical implications. To translate these results to public health policy and clinical practice, a precise understanding of our biological measurements that ensures reproducibility is necessary. Failure to account for differences in protocols can confound results from different experiments and prevent generalizable discoveries [1].

In case-control experiments involving microbiome measurements, the following questions are of interest to epidemiologists in generating further hypotheses about disease mechanisms and treatment:

1. Is there a difference in the $\alpha$-diversity (within-sample diversity) of bacteria observed in samples from cases versus those of controls?

2. Do the observed quantities of bacteria in samples from cases arise from a different multivariate probability distribution than those from controls? If so, which bacteria are found in different quantities in cases versus controls?

Choices in sample processing protocols and bioinformatics protocols for measuring the microbiome can affect the ability to find answers to these questions. Also, differences across labs in these choices can lead to contradictory conclu20 sions.

Labs make different choices in microbiome measurement protocols based on their particular environment of interest. For example, in our measurements of the vaginal microbiome using $16 \mathrm{~S}$ rRNA sequencing, we designed polymerase chain reaction (PCR) primers that were sure to amplify Chlamydia and other key taxa [2, 3]. Doing so makes comparing our results to other studies using different primers difficult, but the ability to detect particular taxa of interest can be a higher priority. Contrary to claims from many microbiome researchers [1], not all labs should use the same protocols for biological measurements, but should aim for the best characterization of the environment and disease(s) of interest.

30 Understanding the effects of different choices in protocols, and publishing details 
of protocols [4], then becomes of paramount importance so that results from multiple studies can be reproduced and assimilated.

Because host-microbiome interactions are dynamic and complex, clear signals are often difficult to detect in case-control studies involving microbiome measurements. Most studies to date focus on only a single 'omic type at a single time point, thereby providing an incomplete picture of the environment. Therefore, a careful treatment of data is important for reproducible results.

I discuss three challenges in conducting reproducible microbiome research for case-control studies. For each, we present the significance of the challenge, evaluate commonly-held views, pose unanswered questions, and suggest strategies for overcoming them. The challenges are:

1. Differences in observed and actual microbial community composition.

2. Detecting "rare" taxa in microbial communities.

3. Choosing properly-powered analysis methods.

45 Normalization methods have been proposed that address one or more of these challenges based on certain assumptions regarding sample processing. Normalization refers to modifications of processed data, and its common use of the term sometimes confounds the three issues. In this commentary, I tease out these challenges, call into question our knowledge regarding typical assumptions for normalization methods, and propose experiments that will provide answers.

I focus here on 16S rRNA surveys, but the principles apply other 'omics technologies. The aim is not to disparage $16 \mathrm{~S}$ technology as unusable. To the contrary, microbiome researchers know the most about $16 \mathrm{~S}$ measurements, and the challenges that I discuss here are likely surmountable. Many of the other ${ }_{55}$ 'omics technologies such as whole metagenome shotgun sequencing (WMGSS), cytokine assays, and untargeted metabolomics suffer from many of the same challenges as 16S rRNA surveys, and yet have additional layers of difficulties that must be addressed.

A common myth in the microbiome research community is that problems 60 with microbiome measurements and differences in choices among protocols can 
Figure 1: Stacked bar plot of the results of five replicates of a defined mock community with bacteria from six genera. The "True" bar reflects the actual mixing concentrations.

only be solved by further advances in technology. The goal of this commentary is to dispel this myth, and demonstrate that many of the challenges in reproducibility of microbiome research can be addressed with current technology.

\section{Differences in Observed and Actual Microbial Community Com- positions}

Sources of Bias. Bias is a difference in observed and actual microbial community compositions. The presence of bias in 16S [5, 6, 17, 8], WMGSS [9, 1], and whole genome amplification (WGA) [10] studies is no secret. Bias refers to differences in the observed and actual quantities in a measurement. In $16 \mathrm{~S}$ rRNA surveys, samples are collected from an environment, filtered from nonorganic material (if needed), subjected to DNA extraction, PCR amplification, sequencing, and taxonomic classification. The end result is a table of counts of DNA fragments (reads) assigned to each taxon or operational taxonomic unit (OTU). Each step in this process can introduce bias and alter the signal so that the observed community composition is different from what is actually there, and affect conclusions drawn from case-control studies.

Evidence for Bias. As an example, consider, the samples in Figure 1. In five replicates, the observed proportions of each bacterium exhibit low variation, but the difference with the true mixing concentration is substantial. Though Fusobacterium species comprised only $20 \%$ of the mixture, the observed proportions were all between 50 and $70 \%$. The observed proportions of Fusobacterium, Prevotella, Lactobacillus, and Staphylococcus were larger than the actual proportions. The observed proportions of Gardnerella and Enterococcus were consistently smaller than the actual proportions. The composition of the positive 85 control mock community is described in the online supplement.

Similar issues arise in the processing of samples for WMGSS, metabolomics 
profiling, and cytokine assays. Compared to these measurement technologies, addressing this issue for 16S rRNA surveys is less complicated because the available reference databases are more complete, sample degradation is typically not an issue, constructing mock communities with known compositions is straightforward, and technical variation is lower.

Current Practices and Open Questions Regarding Bias. Some confuse variation with bias, and mistakenly assume that the small differences between replicates means that there are no problems with bias. Also, control samples of mock communities with a precise quantitation of the bacteria are rare. Quality control is sometimes performed with only environmental replicates. Control experiments are not standardized, and typically are not published.

The current modus operandi is to either use only the presence/absence of bacteria or to simply ignore the issue of bias. A common view is that though the observed abundances in 16S rRNA surveys are distorted, rank abundances are preserved. In other words, if more reads are observed from bacterium A than bacterium $\mathrm{B}$, then there is more bacterium $\mathrm{A}$ than $\mathrm{B}$ in the original sample. In reality, whether rank abundance is preserved or not remains an open question.

Additional open questions regarding bias include

1. How much bias is due to each processing step?

2. Is bias an independent effect for each organism?

3. Can bias be modeled to estimate the true community composition?

We recently published results of an experiment that provides preliminary information about these three questions [8], but does not address the rank abundance issue. The experiment involved processing mixtures consisting of one to seven vaginally-relevant bacteria. By studying mock communities with known compositions, we were able to accurately quantify overall bias. The median total bias for Lactobacillus iners was $38.3 \%$ across the samples in which it was included, meaning that more L. iners was typically observed than was actually present. The median bias for Gardnerella vaginalis was $-30.6 \%$, meaning that less was 
typically observed than was actually present.

By conducting three experiments mixing equal amounts of cells, DNA, and PCR product, and comparing the results, we quantified the contribution to bias of each processing step. We found that DNA extraction and PCR amplification contributed the most to bias, but sequencing and classification contributed very little, indicating that advances in sequencing technology will not address the issue. The median bias due to DNA extraction ranged from $-21.6 \%$ for Atopobium vaginae to $17.6 \%$ for Lactobacillus crispatus. The median bias due to PCR amplification ranged from $-20.8 \%$ for Lactobacillus crispatus to $21.0 \%$ for Lactobacillus iners. For L. crispatus, DNA extraction increases the observed amount while PCR amplification reduces the observed amount for our particular choice of protocols. The median bias due to sequencing and classification was between -5 and $5 \%$ for all bacteria.

The DNA extraction and PCR amplification bias appears to overwhelm possible bias due to $16 \mathrm{~S}$ gene copy number. For example, Streptococcus agalactiae (Group B Strep) had the largest copy number among bacteria in our experiments, yet the bias was negative for each processing step.

Our results also indicate that the presence of certain bacteria can promote or suppress the observed proportions of other bacteria. Special cubic mixture effect models where the true mixing proportion was the response revealed statistically significant interaction terms. An interaction term does not imply a physical interaction between the bacteria, but rather the interaction of the observed amounts between bacteria. For example, when $L$. iners and G. vaginalis were present together in a sample, more $L$. iners and less $G$. vaginalis were observed. As another example, when L. crispatus and G. vaginalis were present in a sample, more $L$. crispatus would be observed than what was actually in the sample.

Finally, we applied models to microbiome measurements from a subject to 145 predict the actual community compositions, and the predicted values matched the clinical diagnosis better than the observed values. The predictive model relied on knowing observed proportions of the seven bacteria that were in the 
mock communities. Whether the same predictions could be made reliably based solely on sequence information remains an open question.

Pitfalls from Bias. The initial results from mock community analyses highlight potential pitfalls in case-control studies. First, in the analysis of casecontrol studies, establishing meaningful effect sizes is dependent on the particular choice of protocols. Assuming that there is sufficient power to detect differences between cohorts, meaningful effect sizes can only be determined by associating microbiome measurements with clinical data. Because the measurements are skewed in different ways by each choice of protocols, a uniform standard is not possible.

Bias can also eliminate the ability to observe roles for bacteria in different cohorts. For example, suppose bacteria A and B play prominent roles in the onset of a disease, but bacterium $\mathrm{C}$ does not. For a choice of protocols, any of the following scenarios could occur:

1. The presence of bacterium A in a sample decreases the amount of B observed, and differences in the amount of bacterium B between healthy and diseased subjects is not discovered.

2. The presence of bacterium $\mathrm{C}$ in a sample decreases the amount of bacterium A observed, and differences in the amount of bacterium A between healthy and diseased subjects is not discovered.

3. The presence of bacterium B in a sample increases the amount of bacterium $\mathrm{C}$ observed. An artificial elevation of the amount of bacterium $\mathrm{C}$ is observed in diseased subjects, leading to a false discovery that bacterium $\mathrm{C}$ has a role in the disease.

Further, different choices of protocols can lead to different false and failed discoveries.

Recommendations for Addressing Bias. Each lab involved in microbiome research should conduct experiments with mock communities to understand the effects of protocol choices. To understand if rank abundance is preserved, multiple mock communities should be checked that are comprised of 
staggered proportions. Replicates of a single control sample cannot capture interactions among bacterial signals properly. Bioinformatic analyses of these experiments are needed to determine the extent to which one can counteract bias from the sequence information alone. That way, generic models can be built that can be applied to adjust the observed amounts of bacteria that are not in the mock communities.

Bias in the proportion of DNA fragments observed from micro-organisms can 
Figure 2: Scatterplot of the number of sequences assigned to taxa not in a mock community versus the library size. A point for each of 172 replicates is plotted. A fitted regression line is depicted with the standard error indicated by shading.

of a sample consisting of a single bacterium, and discovered that at sufficiently low concentrations of the bacterium, mostly contaminants are observed. Some of these contaminants are from the DNA extraction kit. The relationship between concentration/biomass and contamination may be different for different taxa, and is not understood for environmental samples in general. The identification of low-concentration bacteria may be important for disease mechanisms, and their accurate quantification are necessary for comparing the microbiomes of healthy and diseased individuals. Labs should use positive controls to understand how to set a presence/absence cutoff amidst contamination. Further experiments with dilutions of mock communities are needed to understand how "rare" taxa occur.

The counts of contaminants for 172 replicates of a positive control mock community are plotted Figure 2. The composition of the mock community 220 is described in the online supplement. The plot indicates that for samples with sufficient biomass, such as these mock community samples, the level of contamination does not depend on the library size. Therefore, presence and absence can be determined by using a cutoff based on the number of sequences and not the proportion of a sample. For this set of controls, it appears that a 225200 read cutoff would eliminate most of the reads from contaminating taxa.

Once the role of contamination in samples with moderate levels of biomass and those with low biomass is understood, the sequences observed from contaminants can be identified and subtracted from environmental samples [17, 18].

Primer Bias and "Rare" Taxa. Microbiologists correctly interpret the 230 results of $16 \mathrm{~S}$ surveys as compositional data and do not take the number of sequences observed from each bacterium as a measure of biomass. A small number of sequences attributed to a bacterium likely results from a mixture of effects based on sequencing depth, primer bias, and other PCR effects. The 
Figure 3: Stacked bar plot of the results of a mock community processed using different primer sets: P1, P2, a mix of P1/P2, and P192. Two replicates are plotted for each primer set except for P2.

relationship between these three factors is not known, and may be bacteriumdependent. Further, the relationship probably does not satisfy the assumptions, typically those of a probabilistic experiment, used to develop statistical procedures that account for the effects of "rare" taxa. The analogy of sequences being selected as balls from urns should probably be replaced by the analogy of fishing in different tanks with fish having different proclivities for the bait.

As an example of the role of primer bias in the detection of taxa, Figure 3 depicts the observed proportions of bacteria for different primer sets for replicate samples of a mock community. The mock samples had the same composition as that depicted in Figure 1, except that a fixed concentration of Chlamydia was spiked in to each sample. The P1 primer does not amplify Chlamydia at all, and primers P2 and P192 do amplify Chlamydia. A mix of P1 and P2 primers amplifies Chlamydia as well. The choice of primer mix for these mock community samples clearly affects the detection and quantification of Chlamydia.

Chimeras Can Lead to False Positive Detection. Bioinformatics algorithms have been proposed to help identify chimeras which are fused sequences from two or more bacteria [19, 11]. Chimeras can lead to false positive counts of bacteria with sequences whose composition sufficiently matches the observed sequence. The problem of chimera formation is a larger problem for sequence analysis pipelines that rely on a clustering of sequences before taxonomic assignment, commonly known as operational taxonomic unit (OTU) approaches. If instead, sequences are aligned to a reference database, chimeras are identified as mismatches to the database [2]. An ongoing challenge for 16S and other 'omics technologies is the curation of reliable databases for the microbiomes of various human habitats. 
Figure 4: Stacked area plot of bacteria observed in samples taken from the throat and tongue dorsum [20, 21].

\section{Choosing Properly-Powered Analysis Methods}

$260 \quad$ Evaluating Power and Type II Error. New methods for evaluating differences between groups of samples based on taxon sequence counts are often evaluated based on their ability to confirm differences between environments such as body sites. However, we do not know the true type II error rate, nor the maximum possible power, for these experiments with environmental samples. Consider the samples from the throat and tongue depicted in Figure 4 from the NIH Human Microbiome Project [20, 21]. The online supplement contains more information about these samples. Some of the profiles in each environment are very similar to those for the other environment. If we are to assume that the data are sampled from multivariate distributions, then they likely have substantial overlap. Finding a distance measure for ordination or a statistical test that correctly discriminates the throat and tongue samples (or samples from healthy and diseased subjects) can lead us to choose methods that exploit artifacts in our data rather than capture the true patterns. An ideal method would correctly discriminate at a rate based on the (unknown) overlap in the distributions from which the data arise. Power and type II error can be evaluated with simulation studies, but the translation of success in a simulation study to tests for environmental samples depends on how well the assumptions in the distributions of the simulation are satisfied. As discussed in the previous section, the choice of distributions (e.g., zero-inflated negative binomial) often used for simulations of $16 \mathrm{~S}$ data rely on a "drawing balls from urns" analogy that fails to account for bias and contamination.

Power in the Presence of Technical Variation. A challenge that is perhaps overemphasized for $16 \mathrm{~S}$ data is that of technical variation. Technical variation refers to the differences observed when the same sample is processed multiple times. In Figure 1, the differences between replicates are very small. 
Bias is a more troubling issue for distinguishing healthy from diseased subjects based on microbiome profiles.

The concern with technical variation is perhaps a carryover from microarray data analysis, where batch effects required that samples processed at different times be normalized. Refreshingly, this issue does not appear to be a big problem for $16 \mathrm{~S}$ data. For other modern 'omics such as metabolomics and immunoproteomics (cytokines), batch effects are a substantial issue.

There are several remedies for challenges with assessing the power of statistical tests, estimating diversity and richness, and addressing technical variation. A sensitivity analysis involving spiked samples can help to evaluate power. Consider an experiment where (environmental or mock) samples are spiked with new strains at different concentrations. We can evaluate at what concentrations various methods are able to distinguish the new community from the original. Results from these experiments should inform the way we construct simulation experiments to calculate power for statistical tests. These insights should be combined with an understanding of the distributions from which "rare" taxa are drawn to address the effects of PCR on library size and detection.

Technical variation, though a minor issue when compared to bias and taxon detection, is present for repeated microbiome measurements. Technical variation can be measured by processing replicates of environmental and control samples. Adjustments can be then be made to statistical tests to reduce the loss of power from technical variation.

\section{Conclusions}

I reviewed challenges regarding bias, "rare" taxa, and power in microbiome measurements as they apply to case-control studies for human health. To fully address these difficulties, various additional experiments with mock communities are proposed. For bias, calibration experiments using multiple mock communities are proposed. For detecting "rare" taxa, experiments with dilutions of mock communities are proposed. For evaluating the power to discriminate environ- 


\section{Acknowledgments}

My research has been funded in part by NIH Award 8U54HD080784, NIHNIHMD Award 2P60MD002256, and NSF Subaward 5100551 of DMS-1127914. I wish to thank David Edwards for discussions and helpful feedback on drafts of at Virginia Commonwealth University have all contributed to my understanding of the human microbiome.

\section{References}

\section{References}


[2] J. M. Fettweis, M. G. Serrano, N. U. Sheth, C. M. Mayer, A. L. Glascock, J. P. Brooks, K. K. Jefferson, Vaginal Microbiome Consortium, G. A. Buck, Species-level classification of the vaginal microbiome, BMC Genomics 13 (2012) S17.

[3] J. M. Fettweis, J. P. Brooks, M. G. Serrano, N. U. Sheth, P. H. Girerd, D. J. Edwards, J. F. S. III, the Vaginal Microbiome Consortium, K. K. Jefferson, G. A. Buck, Differences in vaginal microbiome in African American women versus women of European ancestry, Microbiology 160 (2014) 2272-2282.

[4] J. Ravel, K. E. Wommack, All hail reproducibility in microbiome research, Microbiome 2 (2014) 8.

[5] S. H. Hong, J. Bunge, C. Leslin, S. Jeon, S. S. Epstein, Polymerase chain reaction primers miss half of rRNA microbial diversity, The ISME Journal 3 (2009) 1365-1373.

[6] A. J. Pinto, L. Raskin, PCR biases distort bacterial and archaeal community structure in pyrosequencing datasets, PLoS ONE 7 (2012) e43093.

[7] J. H. Ahn, B. Y. Kim, J. Song, H. Y. Weon, Effects of PCR cycle number and DNA polymerase type on the 16s rRNA gene pyrosequencing analysis of bacterial communities, The Journal of Microbiology 50 (2012) 1071-1074.

[8] J. P. Brooks, D. J. Edwards, M. D. H. Jr, M. C. Rivera, J. M. Fettweis, M. G. Serrano, R. A. Reris, N. U. Sheth, B. Huang, P. Girerd, Vaginal Microbiome Consortium, J. F. S. III, K. K. Jefferson, G. A. Buck, The truth about metagenomics: Quantifying and counteracting bias in 16s rRNA studies, BMC Microbiology 15 (2015) 66.

[9] A. Wesolowski-Andersen, M. I. Bahl, V. Carvalho, K. Kristiansen, T. Sicheritz-Ponten, R. Gupta, T. R. Licht, Choice of bacterial DNA extraction method from fecal material influences community structure as evaluated by metagenomic analysis, Microbiome 2 (2014) 19. 
[10] A. J. Probst, T. Weinmaier, T. Z. DeSantis, J. W. S. Domingo, N. Ashbolt, New perspectives on microbial community distortion after whole-genome amplification, PLOS ONE 10 (2015) e0124158.

[11] S. M. Huse, D. M. Welch, H. G. Morrison, M. L. Sogin, Ironing out the wrinkles in the rare biosphere through improved OTU clustering, Environmental Microbiology 12 (2010) 1889-1898.

[12] V. Kunin, A. Engelbrektson, H. Ochman, P. Hugenholtz, Wrinkles in the rare biosphere: Pyrosequencing errors can lead to artificial inflation of diversity estimates, Environmental Microbiology 12 (2010) 118-123.

[13] J. N. Paulson, O. S. Stine, H. C. Bravo, M. Pop, Differential abundance analysis for microbial marger-gene surveys, Nature Methods 10 (2013) $1200-1202$.

[14] P. J. McMurdie, S. Holmes, Waste not, want not: Why rarefying microbiome data is inadmissible, PLOS Computational Biology 10 (2014) e1003531.

[15] S. J. Weiss, Z. Xu, A. Amir, S. Peddada, K. Bittinger, A. Gonzalez, C. Lozupone, J. R. Zaneveld, Y. Vazquez-Baeza, A. Birmingham, R. Knight, Effects of library size variance, sparsity, and compositionality on the analysis of microbiome data, PeerJ PrePrints 3 (2015) e1408.

[16] J. C. Lagier, F. Armougom, M. Million, P. Hugon, I. Pagnier, C. Robert, F. Bittar, G. Fournous, G. Gimenez, M. Maraninchi, J. F. Trape, E. V. Koonin, B. L. Scola, D. Raoult, Microbial culturomics: Paradigm shift in the human gut microbiome study, Clinical Microbiology and Infection 18 (2012) 1185-1193.

[17] S. J. Salter, M. J. Cox, E. M. Turek, S. T. Calus, W. O. Cookson, M. F. Moffatt, P. Turner, J. Parkhill, N. J. Loman, A. W. Walker, Reagent and laboratory contamination can critically impact sequence-based microbiome analyses, BMC Biology 12 (2014) 87. 
[18] J. Jervis-Bardy, L. E. X. Leong, S. Marri, R. J. Smith, J. M. Choo, H. C. Smith-Vaughan, E. Nosworthy, P. S. Morris, S. O'Leary, G. B. Rogers, R. L. Marsh, Deriving accurate microbiota profiles from human samples with low bacterial content through post-sequencing processing of Illumina MiSeq data, Microbiome 3 (2015) 19.

[19] B. J. Haas, D. Gevers, A. M. Earl, M. Feldgarden, D. V. Ward, G. Giannoukos, D. Ciulla, D. Tabbaa, S. K. Highlander, E. Sodergren, B. Methé, T. Z. DeSantis, The Human Microbiome Consortium, J. F. Petrosino, R. Knight, B. W. Birren, Chimeric 16S rRNA sequence formation and detection in Sanger and 454-pyrosequenced PCR amplicons, Genome Research 21 (2011) 494-504.

[20] The Human Microbiome Consortium, Structure, function and diversity of the healthy human microbiome, Nature 486 (2012) 207-214.

[21] The Human Microbiome Consortium, A framework for human microbiome research, Nature 486 (2012) 215-221. 


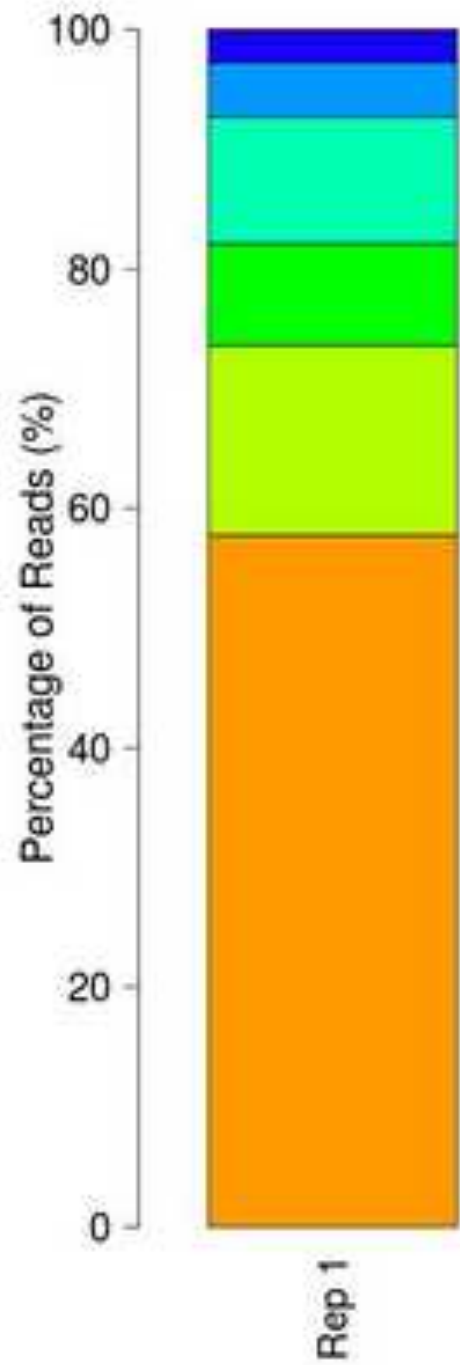

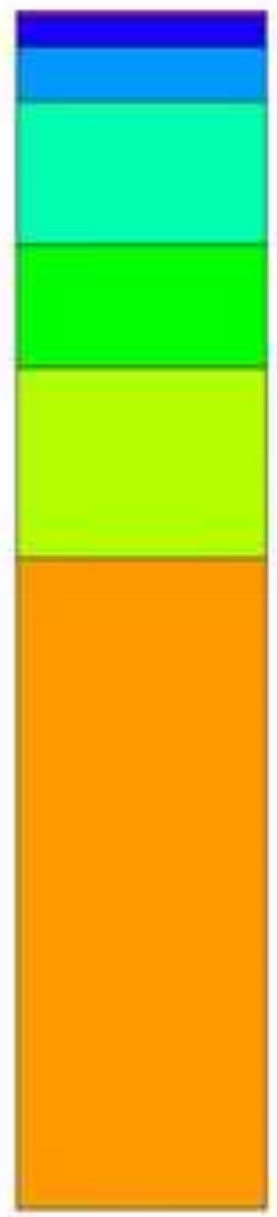

足

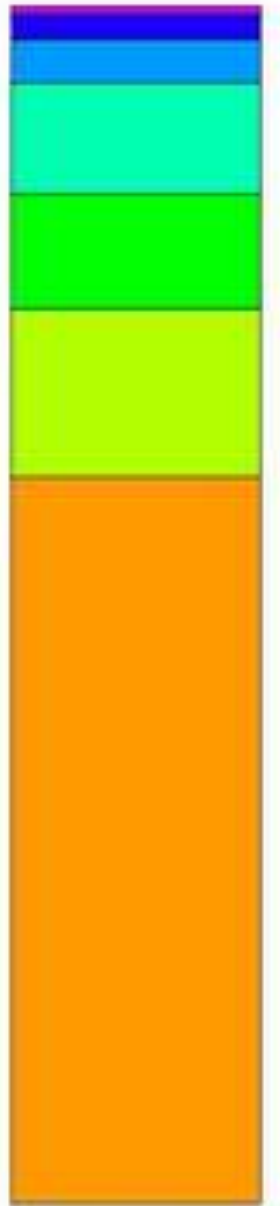

옹

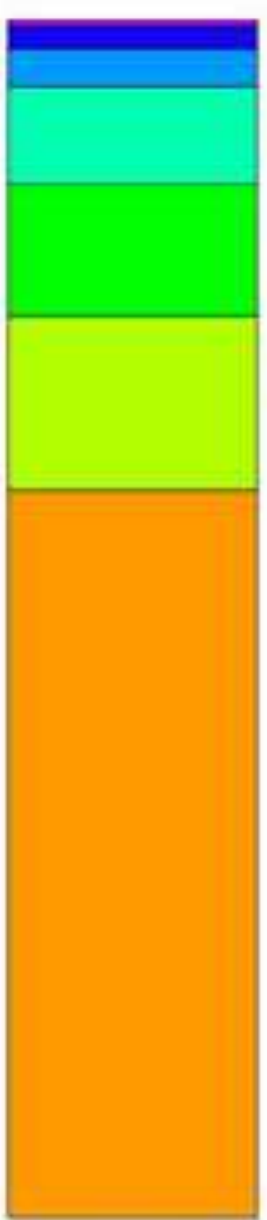

음
옹

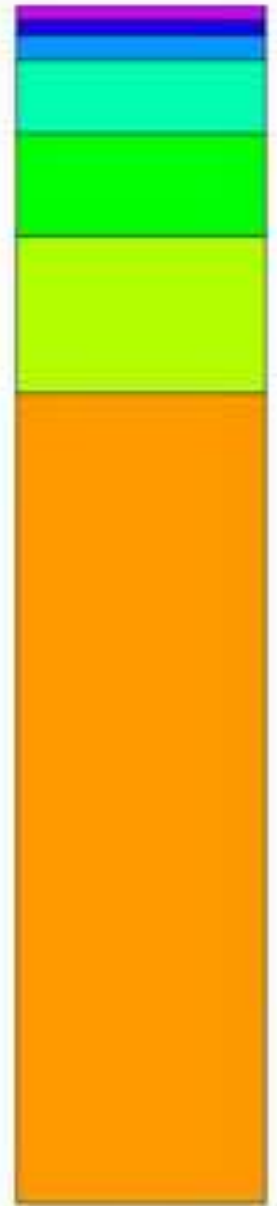

옹

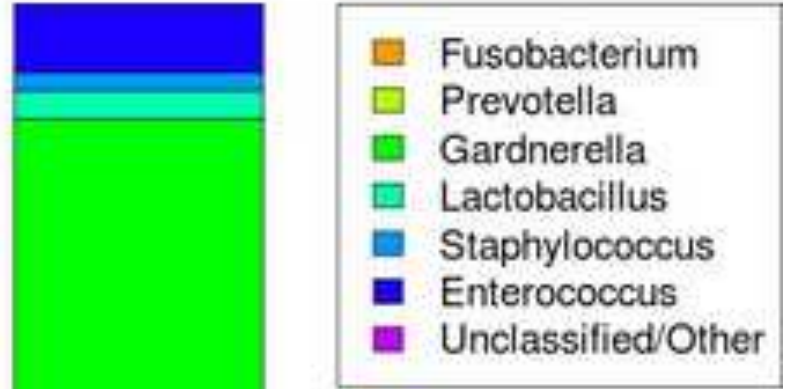

$\stackrel{0}{2}$ 


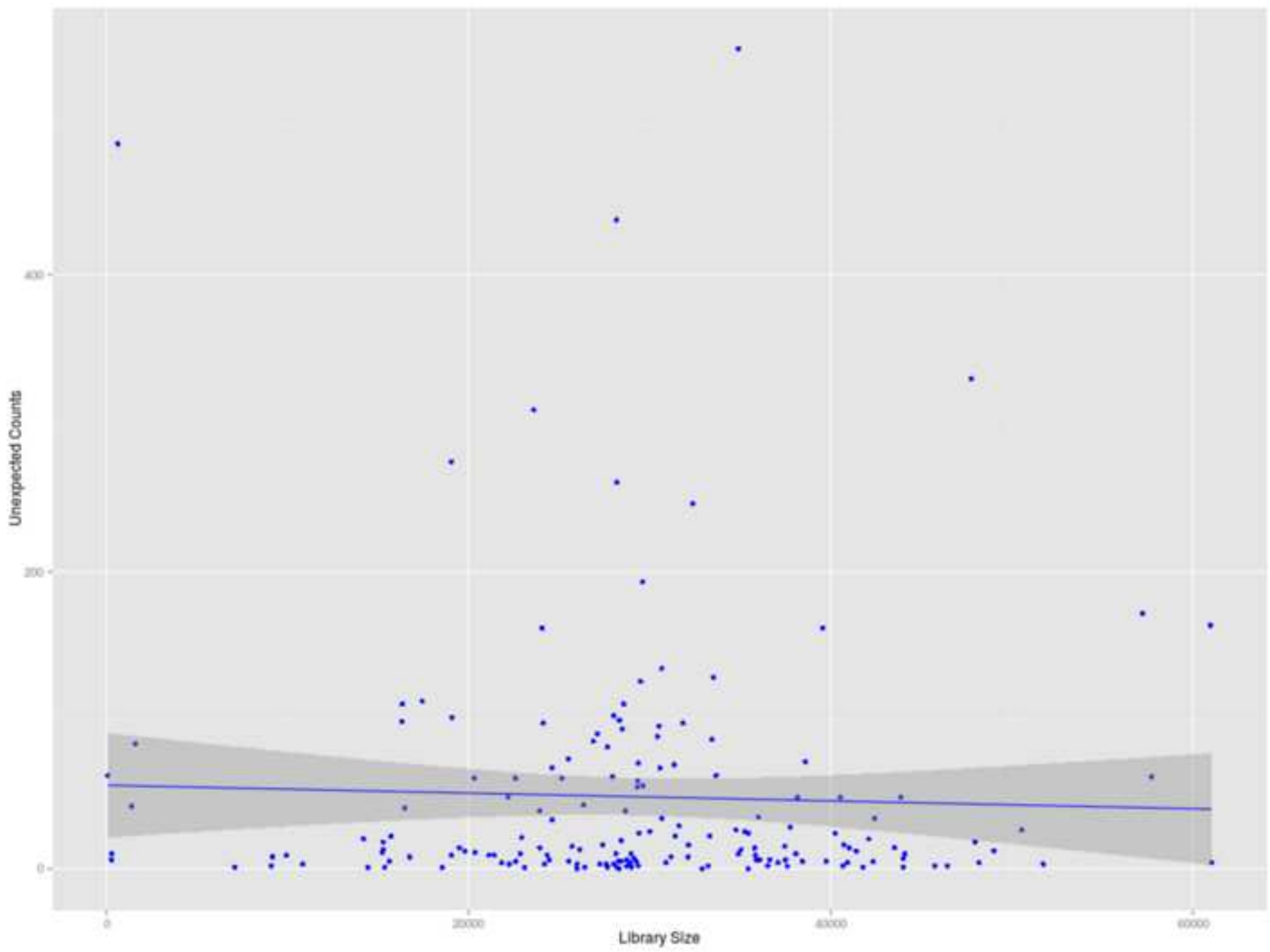



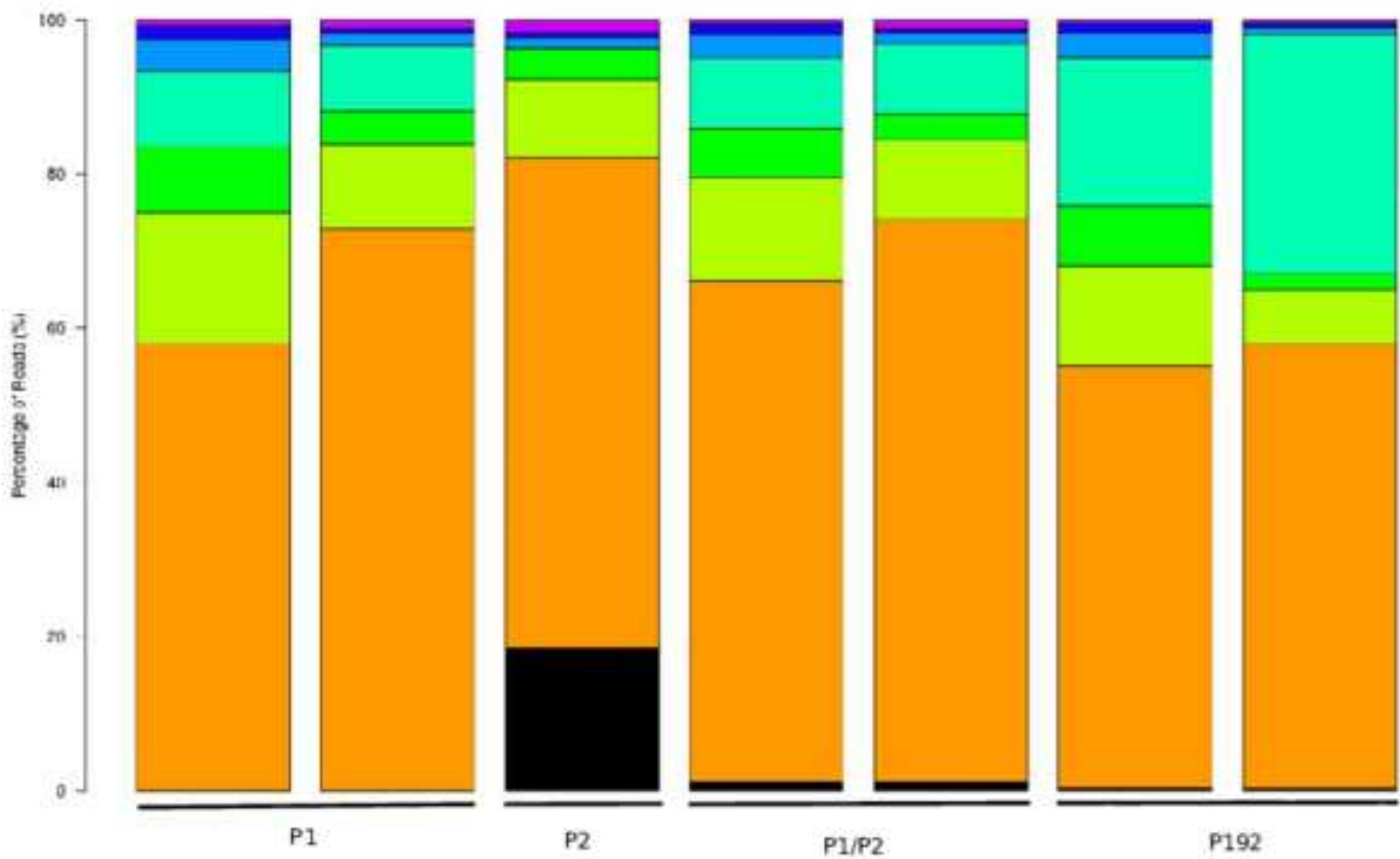

- Cilamela

a r somenderio

7 indarists

a cordterod?

Soptobosectus

- Unciacalred $x$ - 


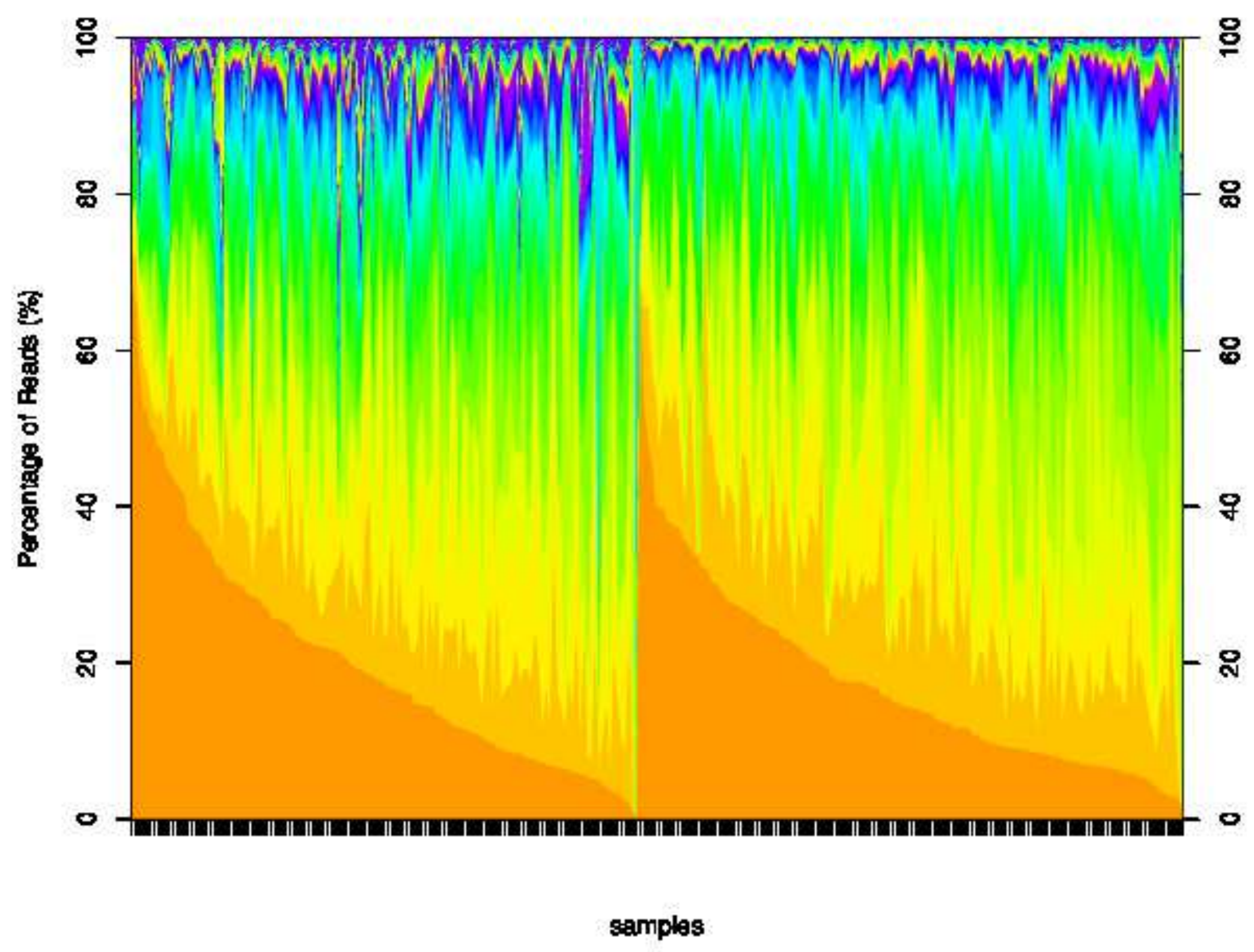

